\title{
Study of the root transcriptome of bread wheat using high-throughput RNA sequencing (RNA-SEQ)
}

\author{
Alexandr Vikhorev \\ Novosibirsk State University \\ Novosibirsk, Russia \\ Institute of Cytology and Genetics \\ SB RAS \\ Novosibirsk, Russia \\ vikhorev@bionet.nsc.ru \\ Elena Khlestkina \\ All-Russian Institute of Plant \\ Recources \\ Saint-Petersburg, Russia
}

\author{
Nikolay Shmakov \\ Institute of Cytology and Genetics \\ SB RAS \\ Novosibirsk, Russia
}

Olesya Shoeva

Institute of Cytology and Genetics SB RAS

Nobosibirsk, Russia

\author{
Anastasia Glagoleva \\ Institute of Cytology and Genetics \\ SB RAS \\ Novosibirsk, Russia
}

\begin{abstract}
Bread wheat (Triticum aestivum L.) is the most important crop in the world. It provides about $20 \%$ of the total calories consumed by humans. For a long time, wheat selection was mainly based on phenotypic traits of the shoot, but the roots were given little attention. As a result, the root system of modern wheat varieties has weakened. Therefore, the study of genetic control of wheat roots development is an urgent issue. In this study, sequencing of RNA libraries from roots and coleoptile of Russian spring variety "Saratovskaya, 29" was performed. De novo transcriptome was assembled. 31,488 up-regulated, 35,851 down-regulated and 18,040 roots-specific transcripts were found. The subsequent analysis of genes with differential expression will allow choosing the candidate genes for development of wheat varieties with resistant root system.
\end{abstract}

Keywords - wheat, roots, transcriptome, RNA-seq

Introduction

In the middle of $20^{\text {th }}$ century dwarf wheat varieties were obtained, and this event was named the "Green revolution". These varieties had strong, short stem, dark-green erected leaves and were able to produce a large amount of yield under nitrogen fertilizers [1]. But growing population size and climate changes require an increase in grain production not only in well-fertilized, but also in nutrient-poor soils. Therefore, there is necessity in second green revolution, and its source can be roots [2].

Hidden underground, roots perform functions crucial for the plant organism life, such as water acquisition, supply of nutrients and anchoring. Despite that, roots were out of attention of breeders throughout the history of cereal plant breeding. This has led to the weakening of the roots of modern varieties compared to their ancestors. Research shows that the root system of modern wheat varieties may not be able to adequately supply water and nutrients for developing seeds [3].

A convenient method to study the genetic control of roots development is transcriptome profiling using high- throughput RNA sequencing (RNA-seq). The method allows us to evaluate gene expression across the entire genome, as well as to find specific genes responsible for roots development that will be used in the future during marker-assisted selection of wheat varieties with resistant root system.
The aim of the current study is revealing differentially expressed genes between the root and shoot transcriptomes of allohexaploid wheat using RNA-seq analysis and identifying root-specific genes involved in the wheat root system development.

\section{Materials and Methods}

\section{Plant material and RNA sequencing}

RNA was extracted from 4-days roots and coleoptiles of seedlings of Russian spring wheat cultivar "Saratovskaya 29" in three biological replicas. One sample contained roots or coleoptile from six different plants. Sequencing was performed using Illumina NextSeq 550 platform in Institute of Cytology and Genetics SB RAS, 75-bp reads was obtained.

\section{In silico analysis of RNA libraries}

De novo assembly of transcriptome was performed using Trinity software. To evaluate quality of obtained transcriptome Transrate software was used. Quantification of reads was performed using Salmon software. To obtain different expressed transcripts edgeR package was used. For functional annotation Transrate, AgriGO and BlastKOALA services were used.

\section{Results}

\section{De novo transcriptome assembly and evaluating}

In our study, for the first time, de novo transcriptome of the Russian spreeing variety "Saratovskaya 29" was assembled. In the de novo assembly, $94.2 \%$ of conserved monocotyledonous plant genes were found, as well as 112,934 open reading frames, which is close to the number of reading frames in the annotated reference transcriptome (119,267 open reading frames). This metrics indicate a high quality of the obtained transcriptome.

\section{Differential expand specifically expressed transcripts}

$67,339(50 \%$ of the total) differentially expressed transcripts were found, of which 31,488 were up-regulated in the roots, and 35,851 were down-regulated. Root-specific expression was found in 18,040 (13\%) transcripts.

\section{Functional annotation}

The analysis of saturation of gene ontology terms revealed that the most common products of differentially expressed 
genes are membrane proteins and proteins associated with phosphorylation. These proteins are probably root-specific receptors and regulatory proteins responsible for root development.

\section{References}

11] G. S. Khush, "Green revolution: The way forward", Nature Reviews Genetics, vol. 2, no. 10. Nature Publishing Group, pp. 815-822, Oct2001

[2] J. P. Lynch, "Roots of the second green revolution", Australian Journal of Botany, vol. 55, no. 5. pp. 493-512, 2007.

[3] J. G. Waines and B. Ehdaie, "Domestication and crop physiology: Roots of green-revolution wheat", Annals of Botany, vol. 100, no. 5. Oxford University Press, pp. 991-998, Oct-2007. 